\title{
Prolonged survival in motor neuron disease: a descriptive study of the King's database 1990-2002
}

\author{
M R Turner, M J Parton, C E Shaw, P N Leigh, A Al-Chalabi
}

J Neurol Neurosurg Psychiatry 2003;74:995-997

Motor neuron disease is a clinically heterogeneous disease with significant differences in survival. The authors have characterised a subset of long term survivors seen in a tertiary clinic over a 12 year period in terms of clinical variables and demographics, comparing them with short term survivors and the remaining population. Thirty of 769 patients survived more than 10 years, corresponding to $4 \%$ of the total population. Significantly younger onset of disease symptoms and a predominance of pure upper motor neuron signs at presentation characterised the long term survivors, but factors traditionally regarded as being associated with poor prognosis were also well represented. For a few people with motor neuron disease there remains the hope, whatever the initial presentation, that their subsequent survival will be longer than expected.

A lthough average survival in motor neuron disease (MND) is widely quoted as 36 months, there is a wide variation in prognosis, and clinical heterogeneity represents a significant hurdle in clinical trials. We have previously examined prognostic factors using a database and demonstrated, using Cox regression analysis, that certain factors can be used to robustly predict individual survival for most patients. ${ }^{1}$ However, a few people survive much longer than expected from such a model. Many specialists will have seen patients survive beyond 10 years, and this possibility is an important source of hope for those with MND. ${ }^{2}$

It is established that phenotypic variants from "classic" amyotrophic lateral sclerosis (ALS) are associated with improved prognosis, for example the "flail arm" presentation ${ }^{3}$ and the upper motor neuron only presentation of primary lateral sclerosis (PLS). ${ }^{4}$ Those with bulbar onset of symptoms, older age at onset and more definite El Escorial category at presentation, have been associated with reduced survival. ${ }^{15}$

In this study, we describe patients with significantly long survival, comparing them with an equal number of the shortest surviving patients and the remaining patients from the King's MND database, in an attempt to explore further the relation between prognostic factors and individual survival.

\section{METHODS}

Patients seen at a tertiary referral MND centre from 1990-2002 were prospectively recorded in a database. Diagnosis was made by two consultant neurologists after exclusion of other conditions, including screening male patients with lower motor neuron presentations for the androgen receptor trinucleotide repeat sequence characterising the Kennedy syndrome. Patients with a diagnosis of amyotrophic lateral sclerosis (ALS), primary lateral sclerosis (PLS), or progressive muscular atrophy were included in the analysis. All patients given, or subsequently found to have, an alternative diagnosis were excluded.
Follow up for survival (defined from symptom onset) was carried out at intervals and date of death recorded. Those lost to follow up were searched for in collated United Kingdom death certificates and through general practitioner contact. Survival was calculated where status was definitely known at the time of censoring (31 December 2001). People with a survival greater than 10 years were designated "long survivors". For comparison, an equal number of "short survivors" were defined from the database using only deceased patients ranked for survival consecutively beginning with the shortest. After removal of the long and short survivor groups, the remaining database patients were treated as a separate population for comparison.

Date of birth, sex, site of onset (bulbar or limb), and date of symptom onset were recorded. The 1994 El Escorial classification was used, ${ }^{6}$ all long survivors having presented before the updated classification. Where recorded, forced vital capacity (FVC) at presentation was noted.

Significance of variables was tested using a two tailed $\chi^{2}$ test or linear regression. Where an expected cell count was less than 5, Fisher's exact test was used. The Bonferroni correction was applied to adjust for multiple comparisons. Calculations were performed using SPSS (version 10.0.7, SPSS Inc, Chicago, IL).

\section{RESULTS}

A total of 985 patients were seen between 1990 and 2002. Survival status was known for 769 (78\%). Overall mean survival was 43 months (SD 34 months; median 39 months; range 3-299 months). Thirty patients with survival greater than 120 months ( $4 \%$ of the total) were used for the subsequent analysis (mean 162 months; SD 45 months).

Of the long survivors, $70 \%$ remained alive, and $60 \%$ were male. The mean age of onset was 43 years (range 20-72; SD 13). Only two of the 30 cases had a family history of MND. Onset was bulbar in 23\% $(n=7)$, limb in $77 \%(n=23 ; 10$ upper limb, 13 lower limb). El Escorial category at presentation was "suspected" in 13\% ( $\mathrm{n}=4)$, "possible" in $40 \% \quad(\mathrm{n}=12)$, "probable" in $23 \%(n=7)$, and "definite" in $23 \%(n=7)$. Five cases ( $17 \%$ ) presented with solely upper motor neuron (UMN) features (four limb, one bulbar onset), compared with $2 \%$ for the remaining database population. Most of the long survivors had mixed signs at presentation (70\%).

Eight of the long survivors received riluzole, one underwent percutaneous gastrostomy, but none received assisted ventilation. FVC at presentation was recorded in 10 long survivors and was $100 \%$ for six, who all had limb onset disease. Three of the remaining four with values less than $100 \%$ had bulbar onset disease.

There was no significant difference in sex, site of onset (bulbar, upper or lower limb), age of onset, or El Escorial category, between the long survivors and the remaining database

Abbreviations: $M N D$, motor neuron disease; $A L S$, amyotrophic lateral sclerosis; PLS, primary lateral sclerosis; FVC, forced vital capacity 
Table 1 Long survivors compared with the remaining database population and short survivor group

\begin{tabular}{|c|c|c|c|c|c|c|}
\hline Variable & Category & LS $(n=30)$ & SS $(n=30)$ & $\operatorname{RP}(n=709)$ & $p L S \vee R P$ & $p L S \vee S S$ \\
\hline \multirow[t]{2}{*}{ Survival at census } & Alive & 21 & 0 & 177 & & \\
\hline & Dead & 9 & 30 & 532 & & \\
\hline \multirow[t]{2}{*}{ Sex } & Male & 18 & 16 & 442 & & \\
\hline & Female & 12 & 14 & 267 & 0.796 & 0.602 \\
\hline \multirow{5}{*}{ El Escorial category } & Definite & 7 & 15 & 185 & & \\
\hline & Probable & 7 & 8 & 270 & & \\
\hline & Possible & 12 & 4 & 174 & & \\
\hline & Suspected & 4 & 3 & 77 & & \\
\hline & Not known & 0 & 0 & 3 & 0.279 & 0.067 \\
\hline \multirow[t]{3}{*}{ Mixed $v$ pure signs } & Mixed & 21 & 27 & 614 & & \\
\hline & LMN only & 4 & 3 & 78 & & \\
\hline & UMN only & 5 & 0 & 17 & $0.001 *$ & 0.056 \\
\hline \multirow{5}{*}{ Site of onset } & $\mathrm{BO}$ & 7 & 10 & 187 & & \\
\hline & UL & 10 & 10 & 265 & & \\
\hline & $\mathrm{LL}$ & 13 & 10 & 255 & & \\
\hline & Trunk & 0 & 0 & 1 & & \\
\hline & Not known & 0 & 0 & 1 & 0.782 & 0.631 \\
\hline Age of onset (mean in years) & & 43 & 62 & 57 & $<0.001$ * & $<0.001^{*}$ \\
\hline
\end{tabular}

population. However, age of onset was significantly lower in the long survivors ( 43 compared with 57 years; $p<0.001$ ), and significantly higher in the short survivors (62 compared with 57 years, $\mathrm{p}=0.029)$, when both were compared with the remaining population.

Table 1 summarises the results for the long survivors, short survivors, and the remaining population, with comparisons between all three groups.

\section{DISCUSSION}

We have characterised a group of long surviving MND patients in an attempt to find clinical/demographic indicators of good prognosis. The number is comparatively small, although it is probably higher than in the general MND population because of the specialist nature of the clinic. Although survival data are $78 \%$ complete there is still the potential for a "healthy" bias in our population. Incomplete follow up could also potentially lead to inclusion of a person with an incorrect diagnosis. Within the long survivors however, who by definition have been followed up for 10 years, this is unlikely. Furthermore, the finding that $4 \%$ of the total database survived beyond 10 years is in keeping with a previous clinic based study.

The most striking finding is that the long survivors are demographically similar to the remaining MND population, apart from being on average 14 years younger at disease onset. On the other hand a late age of onset does not exclude long survival, three people having developed MND in their seventh decade and one in the eighth. Younger age of onset is widely recognised to be associated with longer survival. ${ }^{1578}$ The long survivors may represent a group with a greater genetic contribution overall, as many recessive diseases present at a younger age. Familial ALS has a mean age of onset 10 years younger than for sporadic ALS, ${ }^{79}$ but is not necessarily associated with increased in survival. ${ }^{10}$ The corresponding finding that the short survivors are significantly older than the remaining database population is additional evidence that the age of disease onset in MND is pivotal, and transcends most of the individual variability in survival that can arise using other prognostic factors.

The 3:2 male to female ratio seen in most other studies persists, although an increase in older patients of the female to male ratio, and also bulbar onset disease has been previously noted. ${ }^{8}$ Four of the seven bulbar onset long survivors were women. Although bulbar onset disease is traditionally regarded as a poor prognostic factor, our previous analysis showed that this is not independent of its association with confounding variables such as older age of onset and more definite El Escorial category at presentation. ${ }^{1}$ However, it is still surprising that 7 of the 30 long survivors had bulbar onset, comparable with the $25 \%$ seen in most studies. Furthermore, only one of these patients presented with a pure upper motor neurone syndrome, and unusually one had pure LMN signs at presentation.

Although not designed as a staging criterion, overall database analysis has confirmed previous findings that the El Escorial category at presentation has independent prognostic value, ${ }^{15}$ though this is not a uniform finding. ${ }^{11}$ Presenting with ALS in the definite category is however compatible with survival of more than 10 years. Seven long survivors were in this category, four of whom had bulbar onset disease. One person with bulbar onset, El Escorial definite ALS and onset in the sixth decade remains alive after nearly 20 years. At the other end of the scale, patients presenting with solely upper motor neuron signs were over-represented in the long survivors compared with the overall database (the PLS cases accounting for the comparatively high number of long survivors within the El Escorial possible category at presentation, using original criteria). Strikingly, within the short survivors no cases of pure upper motor neuron signs at presentation were seen, whereas the El Escorial definite category was well represented as might be expected. The short survivors did not differ significantly from the remaining population for any of the clinical variables studied, raising the possibility that unidentified factors may be more influential at this stage of the disease.

Only a few of the long survivors received any interventions, for example, riluzole or percutaneous gastrostomy, suggesting these are not the explanation for the longer survival. Although the data are limited, it is striking that all limb onset patients bar one had maximal FVC readings at presentation. The consistently low readings in the bulbar onset patients are likely to reflect poor lip seal or laryngeal spasm at the time of testing, reinforcing the unsuitability of this test in such patients and the need for a more reliable measure such as sniff pressure. ${ }^{12}$ 
In conclusion, typical ALS with bulbar onset, onset later in life, or in the definite El Escorial category at presentation, does not preclude a long survival, though younger onset and pure upper motor neuron signs at presentation are more likely to confer a better prognosis. For a few people with MND there remains the hope, whatever the initial presentation, that their subsequent survival will be longer than expected.

\section{ACKNOWLEDGEMENTS}

We would like to thank all the patients who have attended the clinic over the past 12 years, and all the staff at King's who have helped to maintain the database during this time.

\section{Financial disclosure}

MRT and PNL have previously received honorariums for speaking from Aventis Pharmaceuticals. PNL has been funded for the conduct of a clinical trial within the department, and has received consultancy fees from Aventis. A Health Economics study within our department was funded by Aventis. The Charcot Young Investigator Prize awarded to AAC by the MNDA on behalf of the International Alliance of ALS/ MND Associations was funded in part by Rhone-Poulenc-Rorer.

\section{Authors' affiliations}

M R Turner, M J Parton, C E Shaw, P N Leigh, A Al-Chalabi, MND Care and Research Clinic, King's College London, UK

Funding: MRT is a Wellcome Trust Clinical Research Training Fellow. MP is funded through a scholarship from The Guarantors of Brain. AAC is funded by a Medical Research Council Clinician Scientist Fellowship. We are most grateful to the Motor Neuron Disease Association (UK) who provide funding for the MND Care \& Research Clinic.

Competing interests: none declared
Correspondence to: Dr A Al-Chalabi, MND Care and Research Clinic, PO4 1 (ANC), Institute of Psychiatry, De Crespigny Park, London SE5 8AF, UK; ammar@iop.kcl.ac.uk

Received 24 September 2002

Accepted in final revised form 25 January 2003

\section{REFERENCES}

1 Turner MR, Bakker M, Sham $P$, et al. Prognostic modeling of therapeutic interventions in amyotrophic lateral sclerosis. Amyotroph Lateral Scler Other Motor Neuron Disord 2002;3:15-21.

2 Grohme K, Maravic MV, Gasser T, et al. A case of amyotrophic lateral sclerosis with a very slow progression over 44 years. Neuromuscul Disord 2001;11:414-16.

3 Hu MT, Ellis CM, Al-Chalabi A, et al. Flail arm syndrome: a distinctive variant of amyotrophic lateral sclerosis. [Letter]. J Neurol Neurosurg Psychiatry 1998:65:950-1.

4 Le Forestier N, Maisonobe T, Piquard A, et al. Does primary lateral sclerosis exist? A study of 20 patients and a review of the literature. Brain 2001;124:1989-99.

5 Chio A, Mora G, Leone M, et al. Early symptom progression rate is related to ALS outcome: a prospective population-based study. Neurology 2002;59:99-103.

6 Brooks BR. El Escorial World Federation of Neurology criteria for the diagnosis of amyotrophic lateral sclerosis. Subcommittee on Motor Neuron Diseases/Amyotrophic Lateral Sclerosis of the World Federation of Neurology Research Group on Neuromuscular Diseases and the El Escorial "Clinical limits of amyotrophic lateral sclerosis" workshop contributors. J Neurol Sci 1994;124 (suppl):96-107.

7 Norris F, Shepherd R, Denys E, et al. Onset, natural history and outcome in idiopathic adult motor neuron disease. J Neurol Sci 1993:1 18:48-55.

8 Eisen A, Krieger C. Epidemiological considerations. In: Amyotrophic lateral sclerosis. Cambridge: Cambriodge University Press 1998:1-30

9 Strong MJ, Hudson AJ, Alvord WG. Familial amyotrophic lateral sclerosis, 1850-1989: a statistical analysis of the world literature. Can J Neurol Sci 1991;18:45-58.

10 Cudkowicz ME, McKenna-Yasek D, Sapp PE, et al. Epidemiology of mutations in superoxide dismutase in amyotrophic lateral sclerosis. Ann Neurol 1997:41:210-21.

11 Traynor BJ, Codd MB, Corr B, et al. Clinical features of amyotrophic lateral sclerosis according to the El Escorial and Airlie House diagnostic criteria: a population-based study. Arch Neurol 2000;57:1171-6.

12 Fitting JW, Paillex R, Hirt L, et al. Sniff nasal pressure: a sensitive respiratory test to assess progression of amyotrophic lateral sclerosis. Ann Neurol 1999:46:887-93.

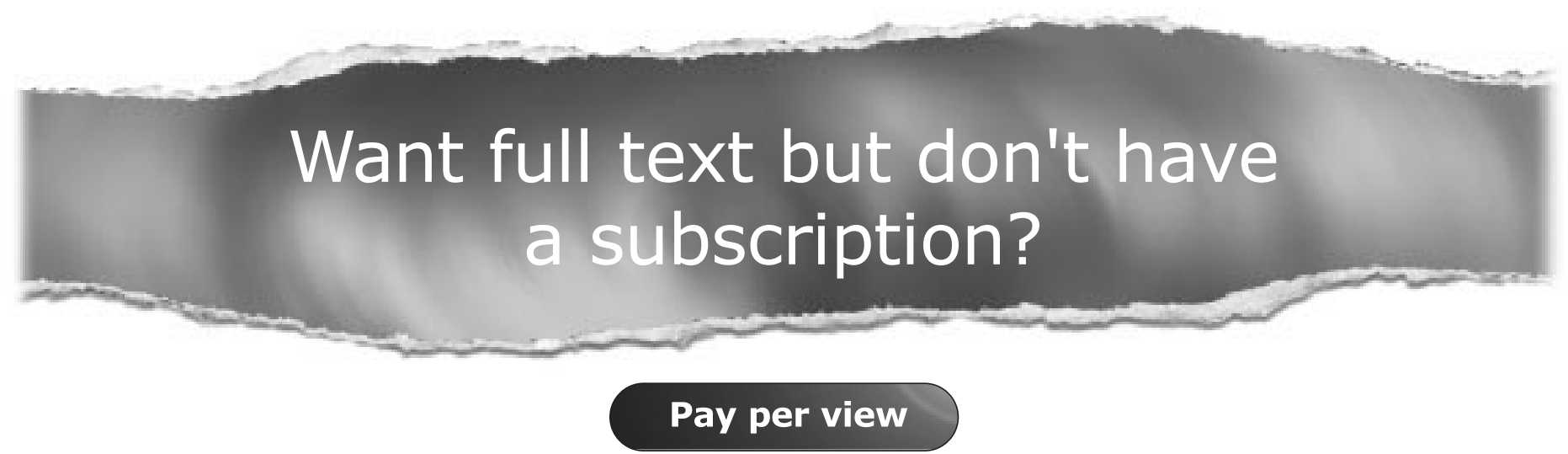

For just $\$ 8$ you can purchase the full text of individual articles using our secure online ordering service. You will have access to the full text of the relevant article for 48 hours during which time you may download and print the pdf file for personal use.

\section{www.jnnp.com}

